\title{
A sun-tracking method to improve the pointing accuracy of weather radar
}

\author{
X. Muth ${ }^{1,2}$, M. Schneebeli ${ }^{2}$, and A. Berne ${ }^{2}$ \\ ${ }^{1}$ Helimap System SA, 1066 Épalinges, Switzerland \\ ${ }^{2}$ École Polytechnique Fédérale de Lausanne (EPFL), School of Architecture, Civil and Environmental Engineering (ENAC), \\ Environmental Remote Sensing Laboratory, 1015 Lausanne, Switzerland
}

Correspondence to: A. Berne (alexis.berne@epfl.ch)

Received: 5 August 2011 - Published in Atmos. Meas. Tech. Discuss.: 29 August 2011

Revised: 5 January 2012 - Accepted: 18 January 2012 - Published: 9 March 2012

\begin{abstract}
Accurate positioning of data collected by a weather radar is of primary importance for their appropriate georeferencing, which in turn makes it possible to combine those with additional sources of information (topography, land cover maps, meteorological simulations from numerical weather models to list a few). This issue is especially acute for mobile radar systems, for which accurate and stable leveling might be difficult to ensure. The sun is a source of microwave radiation, which can be detected by weather radars and used for accurate positioning of radar data. This paper presents a technique based on the similarity between theodolites and radar systems as well as on the sun echoes to quantify and hence correct the instrumental errors which can affect the pointing accuracy of radar antenna. The proposed method is applied to data collected in the Swiss Alps using a mobile $\mathrm{X}$-band radar system. The obtained instrumental bias values are evaluated by comparing the locations of the ground echoes predicted using these bias estimates with the observed ground echo locations. The very good agreement between the two confirms the accuracy of the proposed method.
\end{abstract}

\section{Introduction}

Georeferencing data collected by a weather radar is crucial for the accurate positioning of the processes of interest and for meaningful combination with other sources of data (e.g. topography, land cover, model simulations). Errors in the assumed values of the parameters defining the pointing of a radar have a strong influence on the final uncertainty in the actual location of the collected data. In the case of mobile radar systems, it is of particular importance because radars can be deployed in various (more or less difficult) geographical and climatic contexts. The precise leveling of the radar system may not be easy and can also degrade in time (due for instance to repetitive freeze/thaw or ground instability). In addition, there can be misalignment between the different components of the radar system like the antenna, the arms and the pedestal.

Being a source of radio waves and its position being accurately known and predicted, the sun can be used to estimate the positioning biases affecting a radar system (see Huuskonen and Holleman, 2007, and the references herein). Previous studies have proposed different approaches to do so: for example Arnott et al. (2003) used the sun to estimate the azimuth correction to apply to the data collected by their mobile radar system (mounted on a truck); Darlington et al. (2003) investigated the potential of sun signature in the UK operational radar data, for which the elevation is limited to 4 deg. More recently, Huuskonen and Holleman (2007) and Holleman et al. (2010) further developed this approach for the positioning and the monitoring of operational radar systems by taking into account the effects of the refraction and of the attenuation of the sun signal through the atmosphere.

Another possible approach is to take advantage of strong ground echoes if present in the radar data, either natural (from topography) or man-made (from buildings, towers, antennas, for example). The azimuthal error can then be estimated by minimizing the discrepancy between calculated and observed ground echoes (e.g. Rico-Ramirez et al., 2009).

In terms of radar data positioning, previous studies have so far focused on the estimation of the error in azimuth and elevation. In the present paper, the similarities between the pointing of a radar system and of a theodolite is emphasized. Theodolites have been (and are still) intensively used for land 
surveying since the beginning of the twentieth century, and instrumental errors affecting the positioning of their records have been extensively investigated (e.g. Whyte, 1969; Allan, 2007). The main objective of this work is to transfer existing knowledge and techniques developed for theodolites to weather radar systems. In particular, this means that additional sources of error to azimuth and elevation errors will be considered to improve the accuracy of the pointing of (mobile) radar systems and hence the georeferencing of the collected data.

The paper is organized as follows: Sect. 2 is devoted to the description of the different sources of error in the pointing of a radar system and the similarities to a theodolite. Section 3 details the proposed sun-tracking method, and the results of its application to radar data collected in the Swiss Alps are presented in Sect. 4. Conclusions and perspectives are given in Sect. 5.

\section{Positioning errors in radar systems}

\subsection{Similarities to the theodolite}

In order to determine the position of the data in a given reference coordinate system, the pointing orientation of the electrical axis of the radar antenna has to be known as accurately as possible. It is relevant to emphasize the similarity between the way a radar system (using a mechanically steerable antenna) records the polar position of the data and the way a theodolite does it. Indeed, both polar measurement systems are very similar, recording an azimuthal angle $A$ and an elevation angle $E$ (the terms horizontal and vertical angles are more frequently used in geodesy). An important step is the necessity to orientate the measured azimuthal angles with respect to the cartographic or geographic North (depending on the system chosen for positioning the data). To this purpose, the angle $A_{0}$ between the 0 deg azimuth from the radar and the considered North direction is used. The theodolite and its operating details have been well known for a long time by geodesists and land surveyors. For example Baeschlin (1945), Ollivier (1955) and Whyte (1969) describe the theodolite, in particular its instrumental systematic biases and their effects on azimuthal and elevation angles that are measured. Most of these biases can be modeled and taken into account in the georeferencing process. Similar to a theodolite, a weather radar system can usually observe a target in two different positions of the antenna (assuming that the elevation angle ranges from 0 to $180 \mathrm{deg}$ ). The first position (Position 1 in the following) corresponds to elevation angles smaller than $90 \mathrm{deg}$. The second position (Position 2 in the following) corresponds to elevation angles larger than $90 \mathrm{deg}$, i.e. it refers to the opposite side of the horizontal circle and the complementary value from $180 \mathrm{deg}$ on the vertical circle with respect to Position 1 . This possibility enables the user to detect several instrumental errors which have an
Table 1. Radar positioning errors (in degree).

\begin{tabular}{ll}
\hline Symbol & Description \\
\hline$A_{0}$ & Error in azimuth with respect to the reference North \\
$E_{0}$ & $\begin{array}{l}\text { Error in elevation with respect to the horizontal } \\
\beta_{0}\end{array}$ \\
& $\begin{array}{l}\text { Deviation between the vertical axis of the pedestal } \\
\text { and the vertical }\end{array}$ \\
$\omega_{0}$ & $\begin{array}{l}\text { Orientation of the inclination of the pedestal with } \\
\text { respect to the reference North }\end{array}$ \\
$C_{A_{0}}$ & $\begin{array}{l}\text { Azimuthal collimation error (due to a horizontal } \\
\text { misalignment between the electrical axis and the } \\
\text { arms) }\end{array}$ \\
$C_{E_{0}}$ & $\begin{array}{l}\text { Elevation collimation error (due to a vertical } \\
\text { misalignment between the electrical axis and the } \\
\text { arms) }\end{array}$ \\
\end{tabular}

opposite effect with respect to the measurement position (e.g. azimuthal collimation, the azimuthal component of the angle between the effective electrical axis and the geometrical pointing direction of the antenna).

\subsection{Specificities of radar systems}

The orientation of the electrical axis can be significantly different from the axis of the arms which is the reference for the angular measurement. This is often due to a mechanical imperfection at the interface between the antenna and the arm (problem possibly induced by the mounting/unmounting of the antenna). As a result, it is necessary to adapt the modeling proposed for the theodolite. Table 1 lists the different pointing errors potentially affecting radar positioning that will be considered in addition to $A_{0}$ in the present work. It must be noted that all the azimuthal angles are taken relatively to the reference North in the anti-trigonometric sense. Figure 1 illustrates these errors. The possible azimuthal and elevation eccentricity of the electric axis will be supposed negligible.

Their effects on angular measurements can be derived using spherical trigonometry (see for instance Allan, 2007) and are given in Table 2. The index error $E_{0}$, coming from a possible misalignment between the antenna and the pedestal, has to be distinguished from the vertical collimation error $C_{E_{0}}$ that describes the difference for the vertical orientation between the axis of the arms and the electrical axis. $C_{A_{0}}$ stands for the azimuthal collimation. The inclination of the principal axis, which should ideally be perfectly vertical, is modeled by two angles: $\beta_{0}$ represents the deviation between this axis and the vertical, and $\omega_{0}$ the orientation of the inclination referred to the cartographic North. 

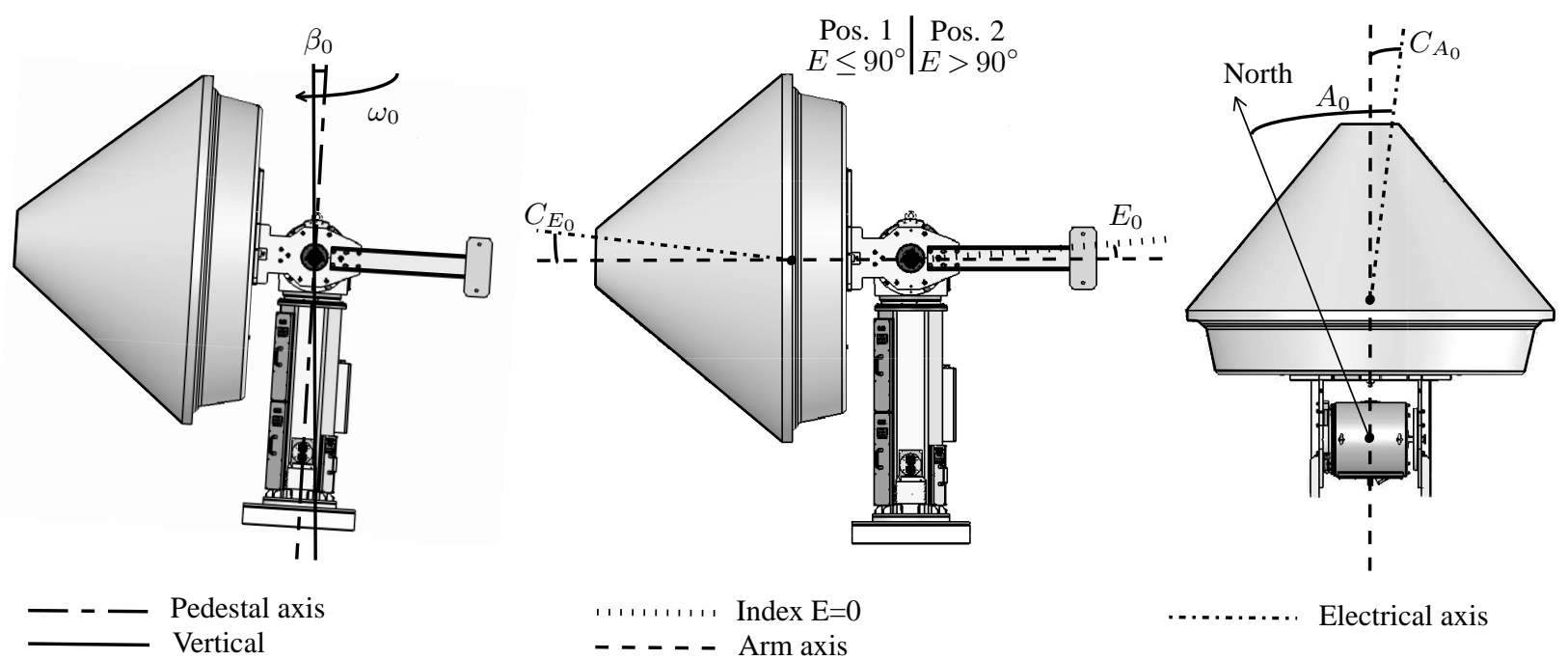

Fig. 1. Main instrumental errors: inclination of the principal axis $\left(\beta_{0}, \omega_{0}\right)$, collimation errors $\left(C_{A_{0}}\right.$ and $\left.C_{E_{0}}\right)$ and the index default affecting the elevation angle $\left(E_{0}\right)$. The antenna drawing has been kindly provided by Prosensing Inc.

Table 2. Modeling of non-negligible instrumental errors.

\begin{tabular}{lccc}
\hline Bias & Effect on the azimuth & Effect on the elevation & Removal \\
\hline Incl. of principal axis & $\beta_{0} \tan (E) \sin \left(A+A_{0}-\omega_{0}\right)$ & $\beta_{0} \cos \left(A+A_{0}-\omega_{0}\right)$ & - \\
Az. collimation & $\pm \frac{C_{A_{0}}}{\cos (E)}$ & - & 2 pos \\
El. collimation & - & $\pm C_{E_{0}}$ & 2 pos \\
Index err. (vert. circle) & - & $E_{0}$ & - \\
\hline
\end{tabular}

\subsection{Atmospheric refraction}

The elevation angle measured by a radar must be corrected for the possible effects of atmospheric refraction (Darlington et al., 2003; Huuskonen and Holleman, 2007). The model proposed by Doviak and Zrnić (1993) gives acceptable corrections in the troposphere depending on the atmospheric conditions (Gao et al., 2008). Nevertheless, another approach has to be considered in the case of solar measurements due to the fact that the sun signal crosses the entire terrestrial atmosphere.

The refraction phenomenon can reach 1 deg for low elevation angles at $\mathrm{X}$ band (see Fig. 2). Considering that the desired accuracy for angular measurements of a radar system is about 0.1 deg (e.g. Huuskonen and Holleman, 2007), the influence of refraction must be taken into account. To do so, the atmosphere is supposed to be homogeneous and stratified. For a standard atmosphere "Mid-Latitude Winter" or "Mid- Latitude Summer", see Kneizys et al., 1996), the refraction index is computed for 1000-m thick layers using the model MPM93 (Liebe et al., 1993). Finally, the SnellDescartes law is applied and the calculation is repeated for the whole range of elevation angles needed. The resulting angular deviation due to refraction is plotted in Fig. 2 and

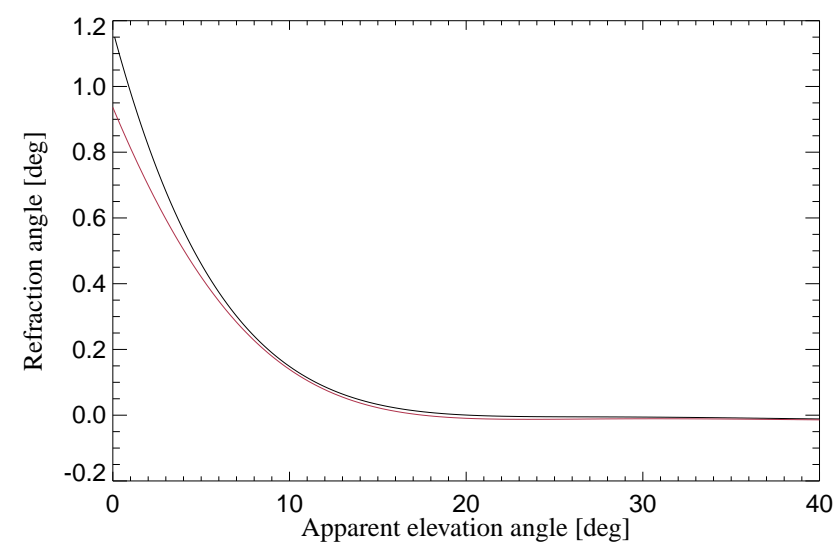

Fig. 2. Refraction model. In black: Mid-Latitude Winter, in red: Mid-Latitude Summer.

has been used to correct the collected sun echoes for atmospheric refraction. In addition, it shows that the atmospheric refraction becomes negligible at high elevations. 


\section{The proposed sun-tracking method}

\subsection{Principle}

Because of the microwave emission of the sun, its signature can be detected in radar data. Knowing the position of the sun and its corresponding record in the polar radar data, the corresponding deviation in azimuth $\left(x_{0}\right)$ and elevation $\left(y_{0}\right)$ can be computed. So during one day, the sun is tracked with successive scanning windows ( $15 \mathrm{deg} \times 5 \mathrm{deg}$, see Fig. 3$)$ alternatively in both positions of the antenna. For each scanning window, the local deviations are computed using the model proposed by Huuskonen and Holleman (2007). A model is then fitted to the these deviations, and its fitted parameter values provide estimates of the instrumental errors. The scanning windows are defined so that the local deviations are monitored for a range of positions on the azimuthal and elevation axes as large as possible in order to have more reliable instrumental error estimates.

\subsection{Processing}

Once the radial containing the sun echo has been detected and stored, the model proposed by Huuskonen and Holleman (2007) is applied to each scanning window. This model is based on the assumption that both the sun emission and the antenna energy pattern have a Gaussian distribution. Because of the conversion of power into $\mathrm{dBm}$, this model takes a quadratic form:

$p(x, y)=a_{1} x^{2}+a_{2} y^{2}+b_{1} x+b_{2} y+c$

$p(x, y)$ is the power measured by the radar (in $\mathrm{dBm}$ ), $x$ and $y$ are the azimuthal and elevation deviations (in deg) between the observed antenna position and the center of the sun, and $a_{1}, a_{2}, b_{1}, b_{2}$ and $c$ are the parameters of the model. These parameters can be estimated by a general least-square technique (Gauss-Helmert for instance, see Grafarend, 2006). From Eq. (1), the azimuthal width $\Delta_{A}$, the elevation width $\Delta_{E}$, the azimuthal deviation $x_{0}$, the elevation deviation $y_{0}$ and the maximum solar power $p_{0}$ can be computed analytically (Huuskonen and Holleman, 2007):

$$
\begin{aligned}
x_{0} & =-\frac{b_{1}}{2 a_{1}} \\
y_{0} & =-\frac{b_{2}}{2 a_{2}} \\
p_{0} & =c-\frac{b_{1}^{2}}{4 a_{1}}-\frac{b_{2}^{2}}{4 a_{2}} \\
\Delta_{A} & =\sqrt{-\frac{40 \log 2}{a_{1}}} \\
\Delta_{E} & =\sqrt{-\frac{40 \log 2}{a_{2}}}
\end{aligned}
$$

where $\log$ denotes the logarithm to base 10 . The values of $\left(x_{0}, y_{0}, p_{0}, \Delta_{A}, \Delta_{E}\right)$ can be obtained analytically using

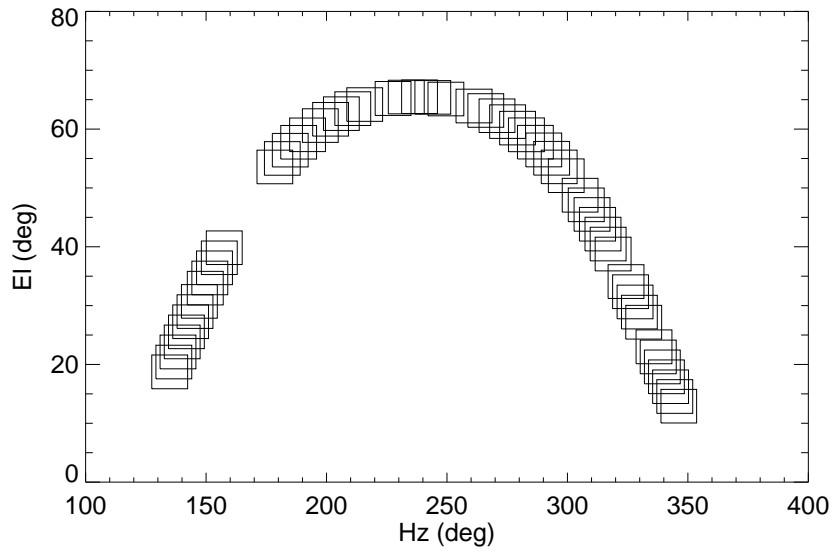

Fig. 3. Successive sun-tracking windows for Position 1, collected on 14 July 2010.

Eqs. (2)-(6), once the the parameters $\left(a_{1}, a_{2}, b_{1}, b_{2}, c\right)$ have been estimated. The detailed derivation of these equations is presented in Appendix A.

The second step consists in fitting the model that takes into account the effects of the most significant instrumental errors (listed in Table 1). This model is composed of 2 error equations relating the azimuthal and elevation deviations to the instrumental errors for the 2 antenna positions (listed in Table 2, see also Allan, 2007):

$\epsilon_{A, i}=\beta_{0} \tan (E) \sin \left(A+A_{0}-\omega_{0}\right)-A_{0}+(-1)^{i-1} \frac{C_{A_{0}}}{\cos (E)}-x_{0}$

$\epsilon_{E, i}=\beta_{0} \cos \left(A+A_{0}-\omega_{0}\right)-E_{0}+(-1)^{i-1} C_{E_{0}}-y_{0}$

where $A$ and $E$ are the considered azimuth and elevation, while $i$ ( 1 or 2 ) indicates the considered antenna position. These equations are based on the assumption that the error terms $\beta_{0}, C_{A_{0}}, C_{E_{0}}$ are small. The "Gauss-Helmert" method is used to estimate the parameters $A_{0}, \beta_{0}, \omega_{0}, C_{A_{0}}, C_{E_{0}}$ and $E_{0}$ from the observed $\epsilon_{A, i}$ and $\epsilon_{E, i}$ values, as well as the associated uncertainties.

If the radar does not have the ability to observe in both antenna positions, we see from Eq. (7) that $A_{0}, \beta_{0}, \omega_{0}$ and $C_{A_{0}}$ can still be estimated, while Eq. (8) shows that only $\left(-E_{0}+(-1)^{i-1} C_{E_{0}}\right)$ can be estimated (which is still very useful for radar data positioning), but not any more $E_{0}$ and $C_{E_{0}}$ separately.

It is assumed in Eqs. (7) and (8) that the possible nonlinearities in the azimuth and elevation angle measurements (from the radar pedestal) are negligible. Significant nonlinearities would affect the estimates of the instrumental errors and their uncertainties, obtained from Eqs. (7) and (8). In order to rigorously take into account such non-linearities, the terms $A_{0}$ and $E_{0}$ should be considered as functions of the azimuth $A$ and the elevation $E$ rather than constant. 
Table 3. Principal technical parameters of the MXPol radar.

\begin{tabular}{ll}
\hline Parameter & MXPol \\
\hline Frequency & $9.41 \mathrm{GHz}$ \\
Polarization & Sim. H and V \\
3-dB beam width & $1.45 \mathrm{deg}$ \\
Range resolution & $15-150 \mathrm{~m}$ \\
Manufacturer & ProSensing, USA \\
\hline
\end{tabular}

\subsection{Accuracy of the parameters}

In order to properly quantify the instrumental errors, it is necessary that the accuracy of the local deviation estimates is significantly smaller than the considered errors. The first step in checking this concerns the quality of the sun ephemeris. The algorithm proposed by Grena (2008) provides an accuracy for the sun position in azimuth and elevation with a maximum error of about $0.003 \mathrm{deg}$ from 2003 to 2023, which is far high enough. In order to reach this accuracy, the geographic position (referred to WGS84 ellipsoid) of the center of rotation of the antenna has to be known with an error smaller than $0.001 \mathrm{deg}$ (i.e. about $110 \mathrm{~m}$, reachable with a standard GPS beacon). It must be noted that the vertical deflection can be non-negligible, especially in mountainous regions, and in that case must be taken into account. This angle represents the difference at the same position between the true zenith, which is the reference for the astronomical measurements, and the normal to the reference ellipsoid considered for the ephemeris (Bomford, 1980). Overall, the accuracy of the inputs is sufficient to estimate the instrumental errors with the desired accuracy $(0.1 \mathrm{deg})$.

\subsection{Data acquisition}

During data acquisition, it is more convenient to use radar in receiving mode only. If this configuration is not available, it is still possible to adapt the acquisition configuration in order to reduce the unwanted effect of the ground and meteorological echoes, by extending the acquisition period and by shortening the pulse length. Collecting the sun's signature during dry weather is the easiest way to avoid such adverse effects if the radar cannot work in receiving mode only. Finally, the scanning procedure has to be accurately synchronized in time (about $1 \mathrm{~s}$ ) to avoid additional uncertainty.

\section{Application to radar data collected in the swiss Alps}

\subsection{Field campaign}

Between September 2009 and July 2011, an X-band Doppler dual-polarization weather radar (named MXPol in the following) was deployed near Davos, Switzerland, at about $2130 \mathrm{~m}$ of altitude (see Fig. 4). The principal technical

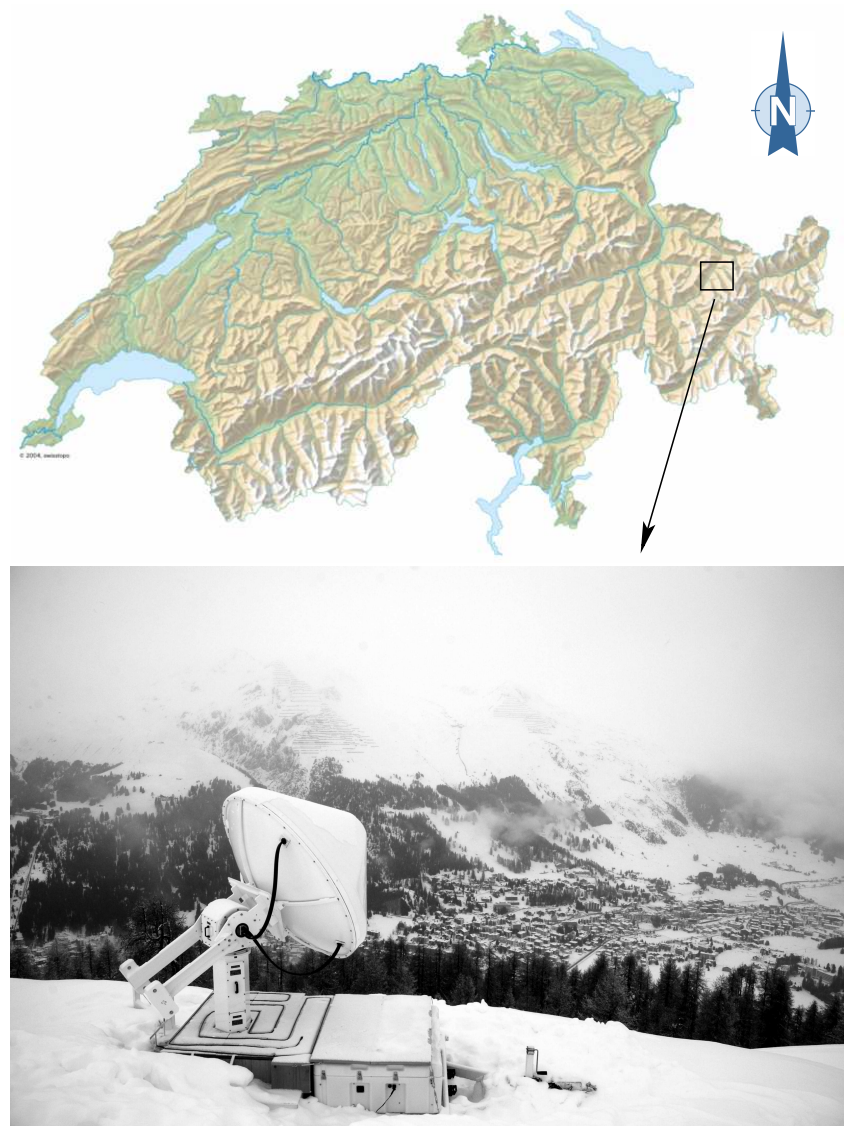

Fig. 4. Radar deployment in Davos (Switzerland). Swisstopo has provided the map of Switzerland in the upper panel.

parameters of the radar system are provided in Table 3. The radar is mounted on a trailer, which was leveled at the installation. But because of the difficult conditions (repetitive freezing/thaw of the terrain, variable load due to the amount of snow), the inclination of the principal axis of the trailer can have changed in time. This issue motivated the development of the proposed technique. In addition, the mountainous context results in a lot of ground echoes in the radar data, which will be used to evaluate the correction derived from the proposed sun-tracking technique. To illustrate the capabilities of the proposed technique, radar data (sun's signature) collected during 2 dry days in July 2010 have been analyzed.

\subsection{Results}

Figures 5 and 6 present the evolution of the azimuthal $\left(x_{0}\right)$ and elevation $\left(y_{0}\right)$ deviations between the theoretical position of the sun and the polar radar record as functions of the azimuthal angle, for the two antenna positions. The instrumental errors are estimated by fitting (in the least-square sense) the models given in Eqs. (7)-(8) to the observed azimuthal and elevation deviations. The obtained values are listed in Table 4. Given these values and the associated uncertainties 


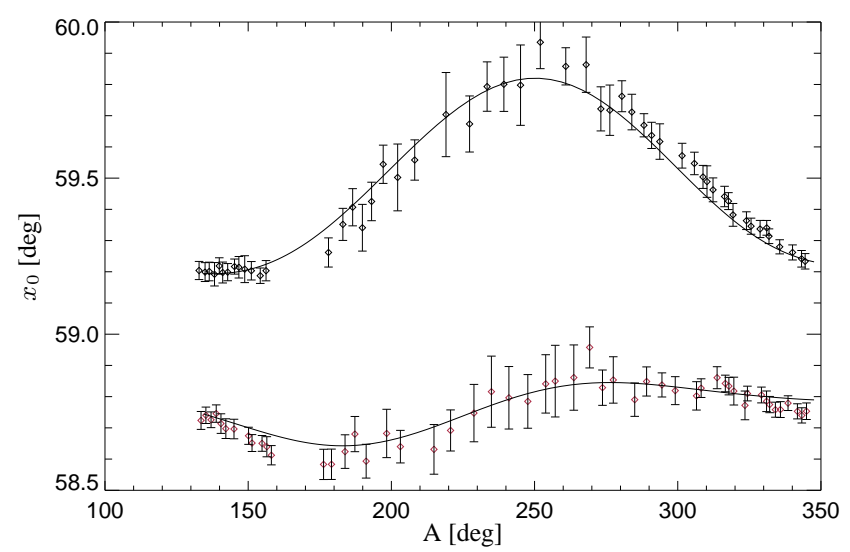

Fig. 5. Evolution of the local azimuthal deviations with respect to the azimuthal angle. The upper points (black diamonds) correspond to Position 1 of the antenna, the lower points (red diamonds) to Position 2. The error bars figure the $99 \%$ confidence interval. The solid lines indicate the fitted models from Eq. (7).

Table 4. Estimated values of the instrumental errors affecting MXPol during the campaign in the Swiss Alps.

\begin{tabular}{lll}
\hline Error & $\begin{array}{l}\text { Estimate } \\
(\mathrm{deg})\end{array}$ & $\begin{array}{l}\text { Uncertainty } \\
(\mathrm{deg})\end{array}$ \\
\hline$A_{0}$ & 300.94 & \pm 0.012 \\
$E_{0}$ & 0.38 & \pm 0.011 \\
$\beta_{0}$ & 0.23 & \pm 0.008 \\
$\omega_{0}$ & 89 & \pm 2.6 \\
$C_{A_{0}}$ & 0.207 & \pm 0.006 \\
$C_{E_{0}}$ & 0.57 & \pm 0.009 \\
\hline
\end{tabular}

(quantified as the standard deviations of the respective parameter estimates), the instrumental errors appear to be significant, and in particular the azimuthal and elevation collimation terms $\left(C_{A_{0}}\right.$ and $\left.C_{E_{0}}\right)$ which cannot be estimated using sun-tracking methods previously published in the literature. The uncertainty associated with $\omega_{0}$ is larger with respect to the others. This is explained by the geometrical function of this parameter which has a secondary impact on the result compared to $\beta_{0}$. So $\omega_{0}$ has a smaller influence on the final correction added to the raw measurements. It can be noted that the accuracy of the obtained error estimates is much lower (except for $\omega_{0}$ but this has limited effect) than $0.1 \mathrm{deg}$, showing the potential of the proposed method.

\section{Evaluation using ground echoes}

\subsection{Principle}

Because of the surrounding mountains, there were many ground echoes in the area covered by MXPol (see Fig. 7). Considering the pointing parameters previously estimated,

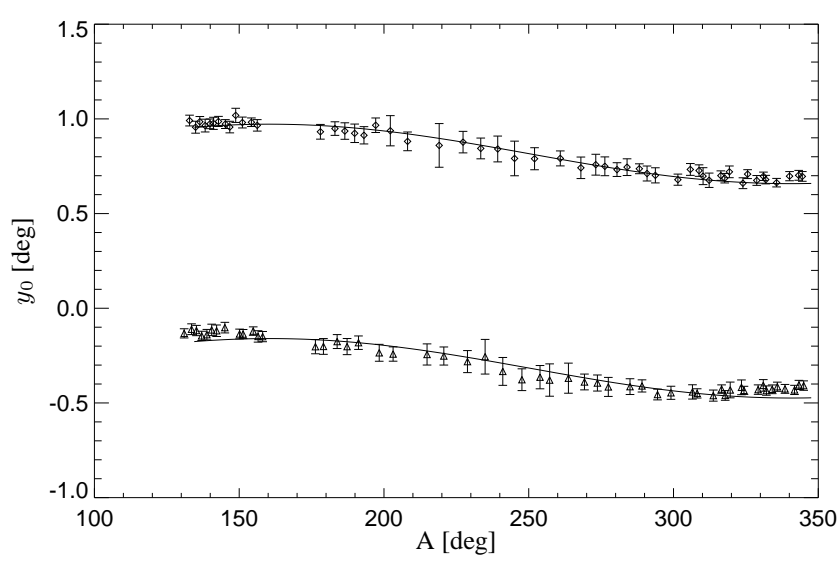

Fig. 6. Evolution of the local elevation deviations with respect to the azimuthal angle. The upper points (diamonds) correspond to Position 1 of the antenna, the lower points (triangle) to Position 2. The error bars figure the $99 \%$ confidence interval. The solid lines indicate the fitted models from Eq. (8).

the detected ground echoes are projected in the Swiss planimetric (MN03) and altimetric (NF02) coordinate systems. Hence, the altimetric deviation with the Digital Elevation Model (DEM) provided by the Swiss Federal Office of Topography (Swisstopo) can be computed. Knowing the accuracy and the resolution of the DEM, the analysis of these differences makes possible the evaluation of the quality of the obtained instrumental error estimates. It must be noted that the possibility to get such a large number of ground echoes at various elevations is specific to this deployment in a mountainous context and is not frequent.

\subsection{Data acquisition}

The data acquisition is performed for both positions of the antenna and is composed of a set of horizontal scans. In order to make the result as sensitive as possible to any systematic bias (especially $C_{A_{0}}$ because its influence directly depends on the elevation), ground echoes must be collected over a significant range of elevations. The acquisition lasted several hours in both positions. In the case of the Davos campaign, grounds echoes were visible and significant from -1 to $8 \mathrm{deg}$ in elevation and over a range larger than $180 \mathrm{deg}$ in azimuth.

\subsection{Creation of a DEM}

The purpose of the post processing is to build a DEM from the ground echoes in these radar data. This DEM can then be compared with a reference one. Here, in the Swiss context of Davos, the 25-m resolution DEM produced by Swisstopo is taken as reference. The accuracy of this product has been tested by Swisstopo and the uncertainty does not exceed $\pm 5 \mathrm{~m}$ in the region around Davos (Swisstopo, 2004). 


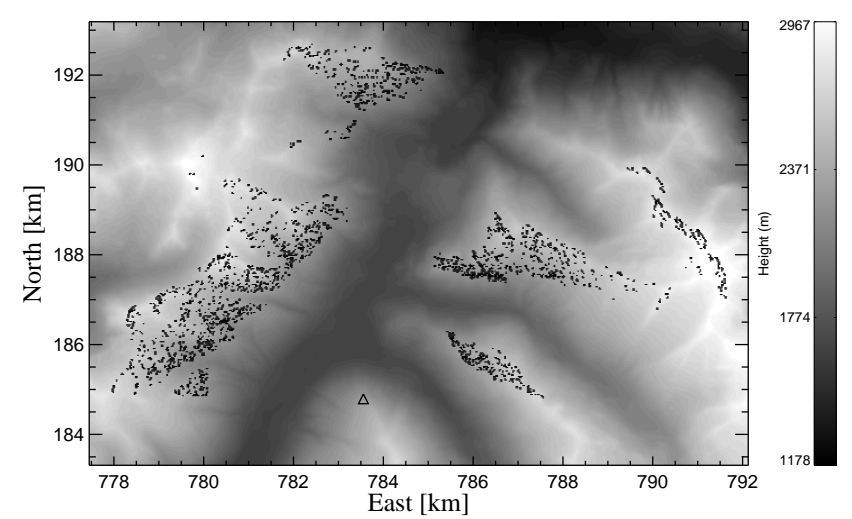

Fig. 7. Projected ground echoes, superimposed on the DEM provided by Swisstopo (Swiss projection system MN03). The radar location is indicated by the triangle.

First, the recorded profiles which have hit the ground are detected by using a threshold which takes the decrease of radar power density with distance into account. The angle of incidence of the radar beam and the type of illuminated surface mainly contribute to the estimation of this threshold (e.g. Ulaby et al., 1986; Rees and Steel, 2001). The polar coordinates of those echoes are then transformed into the Swiss projection system which is also used for the reference DEM. During this step, it is possible to correct the position of the ground echoes for the instrumental errors estimated by the proposed sun-tracking technique. Finally, a grid similar to the Swisstopo one (cell size and positions) can be generated. A null value is affected to the cells which are not covered by any recorded ground echoes.

\subsection{Results}

As both DEMs have the same characteristics, they can be compared and it is possible to analyze the altimetric discrepancies between them in order to evaluate the quality of the estimates of the positioning parameters. Figure 8 presents the distribution of these deviations before and after correction of the positioning errors, for the 2 antenna positions. Figure 8 clearly shows that there is a large improvement when correcting the positioning errors, as indicated by the decrease in the mean difference of the altitudes between the 2 DEMs (from about 90 down to $5 \mathrm{~m}$ ), as well as by the closeness of this mean difference (after correction) for the 2 antenna positions. There is, in addition, a clear decrease in the width of the distributions after correction (the standard deviation decreases about $50 \%$ for Position 1 and about $30 \%$ for Position 2).

To complement the analysis of the distribution of the difference between the 2 DEMs, the spatial correlation of this difference is also investigated by estimating its variogram (e.g. Chilès and Delfiner, 1999; Goovaerts, 2000). Figure 9 shows the experimental variograms before and after
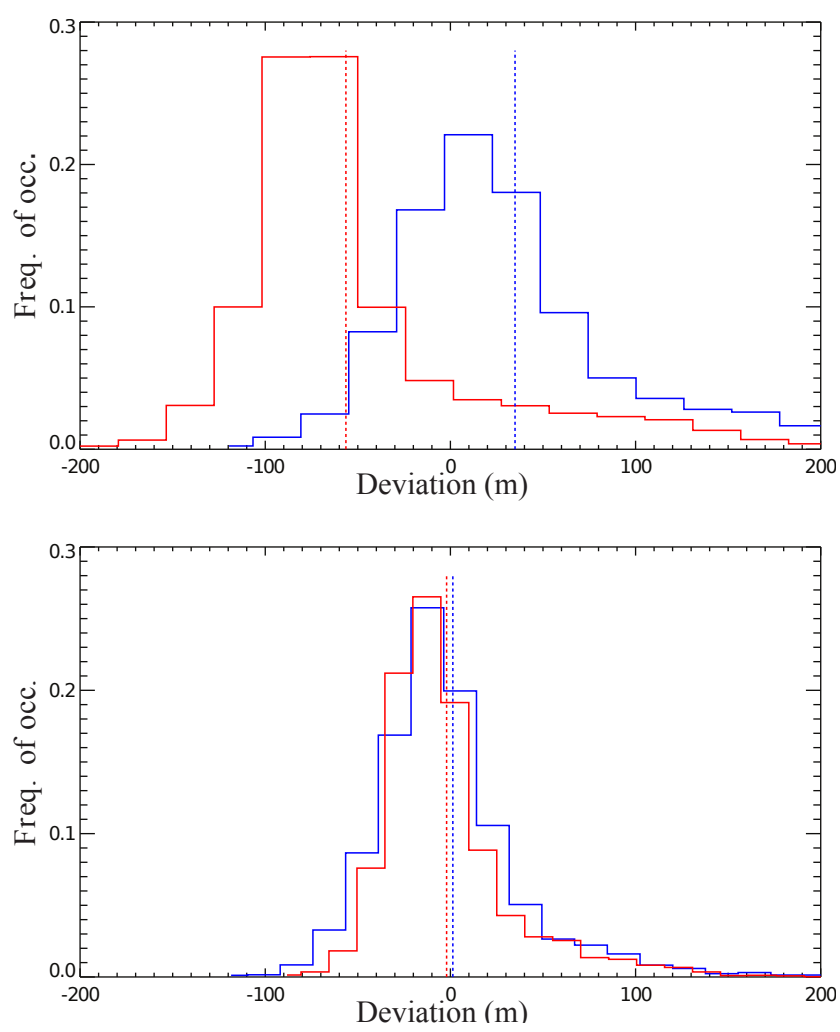

Fig. 8. Histogram of the altimetric deviations between the projected ground echoes and the reference DEM provided by Swisstopo: before pointing correction (top) and after (bottom). The red histogram corresponds to Position 1, the blue one to Position 2. The vertical dashed lines indicate the mean values.

correction for Position 1 of the antenna (the results are similar for Position 2). There is a significant decrease in the variogram values and in its slope after correction, indicating a much lower variability of the difference in altitude between the created and the reference DEM. By showing that the error is lower and is much less spatially correlated, the analysis of the variogram of the discrepancies between the 2 DEMs confirms the analysis of their distributions and the large improvement when using the corrected positioning parameters.

\section{Conclusions}

Weather radar monitors precipitation at long ranges. The accurate positioning of its measurements is hence of primary importance, in particular for the combination with other sources of information. Various sources of error can bias the positioning of the radar, and a new method to correct these sources of error is proposed in the present article, taking advantage of the similarities between radar systems and theodolites (commonly used for a long time in land surveying), as well as of the fact that the sun is a source of microwaves. In addition to the errors in azimuth and elevation, which are 


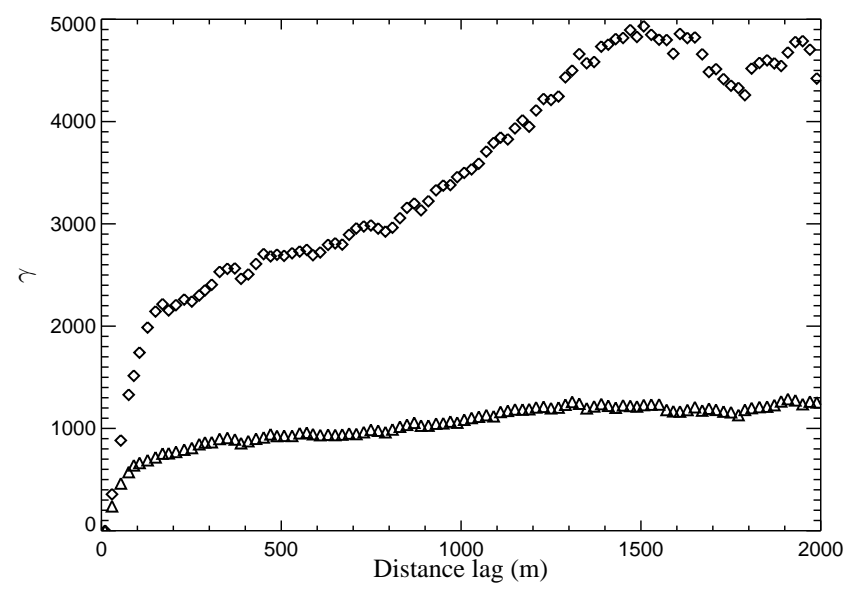

Fig. 9. Variogram of the deviation with respect to the reference DEM: before pointing correction (diamonds) and after (triangles), for Position 1 of the antenna.

usually identified in previously proposed methods, the different positioning errors due to the possible misalignment of the electrical axis with the geometrical axis of the antenna can be automatically quantified. The proposed method is tested on data collected in the Swiss Alps using a mobile X-band radar. The mountainous context enables the creation of a radar digital elevation model, which can be compared to a reference one in order to evaluate the quality of the positioning correction. The large improvement in the agreement between the 2 DEMs shows the reliability of the proposed correction method.

This method is simple and can be applied to fixed or mobile radar systems. The main limitation is the need to follow the sun over a large range of elevation angles to obtain reliable estimates of the different error parameters.

\section{Appendix A}

Derivation of Eqs. (2)-(6)

This appendix presents the detailed derivation of Eqs. (2)(6). $x_{0}$ and $y_{0}$ correspond to the polar coordinates of the maximum power $p_{0}$. The partial derivatives of the power $p$ (given by Eq. 1) with respect to $x$ and $y$ are:

$$
\begin{aligned}
& \frac{\partial p}{\partial x}=a_{1} x+b_{1} \\
& \frac{\partial p}{\partial y}=a_{2} y+b_{2}
\end{aligned}
$$

These derivatives are null at $x_{0}$ and $y_{0}$, which leads to:

$$
\begin{aligned}
& x_{0}=\frac{-b_{1}}{2 a_{1}} \\
& y_{0}=\frac{-b_{2}}{2 a_{2}}
\end{aligned}
$$

Injecting Eqs. (A1) and (A2) in Eq. (1) gives:

$p_{0}=c-\frac{b_{1}^{2}}{4 a_{1}}-\frac{b_{2}^{2}}{4 a_{2}}$

The azimuthal width $\Delta A$ is defined as the width of the power distribution when the power is half the peak power $p_{0}$ in the azimuth. This can be written

$p\left(x_{w i}, y_{0}\right)=p_{0}-10 \log (2)$

where $i=1,2$ (there are $2 x$ values at which the power is half the peak power), and log stands for log in base 10. $\Delta A$ is then quantified as

$\Delta A=\left|x_{w 2}-x_{w 1}\right|$

$x_{w 1}$ and $x_{w 2}$ are derived as follows:

$p\left(x_{w i}, y_{0}\right)=a_{1} x_{w i}^{2}+b_{1} x_{w i}+a_{2} y_{0}+b_{2} y_{0}+c$

Injecting Eq. (A2) in Eq. (A4) results in

$a_{1} x_{w i}^{2}+b_{1} x_{w i}+\frac{b_{1}^{2}}{4 a_{1}}+10 \log (2)=0$

Solving this simple 2-order polynomial equation leads to

$x_{w 1}=\frac{b_{1}}{2 a_{1}}-\frac{b_{1}-\sqrt{b_{1}-4 a_{1}\left(\frac{b_{1}^{2}}{4 a_{1}}+10 \log (2)\right)}}{2 a_{1}}$
$x_{w 2}=\frac{b_{1}}{2 a_{1}}-\frac{b_{1}+\sqrt{b_{1}-4 a_{1}\left(\frac{b_{1}^{2}}{4 a_{1}}+10 \log (2)\right)}}{2 a_{1}}$

Finally, we obtain Eq. (5) by injecting Eqs. (A8) and (A9) in Eq. (A5):

$$
\begin{aligned}
\Delta A & =x_{w 2}-x_{w 1} \\
& =\sqrt{\frac{-40 \log (2)}{a_{1}}}
\end{aligned}
$$

Equation (6) giving the value of the elevation width $\Delta E$ is obtained in a similar way by solving $p\left(x_{0}, y_{w i}\right)=p_{0}-$ $10 \log (2)$.

Acknowledgements. The help of Nicholas Dawes, Marc Ruesch, Dani Lussi, and many others at the Swiss Federal Institute for Snow and Avalanche Research (WSL-SLF), as well as Vali Meier and his team at the Davos-Klosters ski resort is greatly acknowledged, as well as the financial support from the Swiss National Science Foundation (grant 200021-125064).

Edited by: F. S. Marzano 


\section{References}

Allan, A. L.: Principles of geospatial surveying, Whittles Publishing, Caithness, Scotland, UK, 2007.

Arnott, N. R., Richardson, Y. P., Wurman, J. M., and Lutz, J.: A solar alignment technique for determining mobile radar pointing angles, in: Proc. 31st AMS Conf. Radar Meteorol., p. 3, Chapter 12, Seattle, USA, 2003.

Baeschlin, C.: Der Theodolit, Auszug aus der Vorlesung "Vermessungskunde", Buchdruckerei Fluntern, Zurich, Switzerland, 1945.

Bomford, G.: Geodesy, Clarendon Press, Oxford, 4th Edn., 1980.

Chilès, J.-P. and Delfiner, P.: Geostatistics: Modeling spatial uncertainty, Probability and statistics, Wiley, 1999.

Darlington, T., Kitchen, M., Sugier, J., and de Rohan-Truba, J.: Automated real-time monitoring of radar sensitivity and antenna pointing accuracy, in: Proc. 31st AMS Conf. Radar Meteorol., p. 7B.6, Seattle, USA, 2003.

Doviak, R. and Zrnić, D.: Doppler radar and weather observations, 2nd Edn., Academic Press, San Diego, 1993.

Gao, J., Brewster, K., and Xue, M.: Variation of radio refractivity with respect to moisture and temperature and influence on radar ray path, Adv. Atmos. Sci., 25, 1098-1106, doi:10.1007/s00376008-1098-x, 2008.

Goovaerts, P.: Geostatistical approaches for incorporating elevation into the spatial interpolation of rainfall, J. Hydrol., 228, 113-129, 2000.

Grafarend, E. W.: Linear and nonlinear models: fixed effects, random effects, and mixed models, Gruyter, 2006.

Grena, R.: An algorithm for the computation of the solar position, Sol. Energy, 82, 462-470, doi:10.1016/j.solener.2007.10.001, 2008.

Holleman, I., Huuskonen, A., Kurri, M., and Beekhuis, H.: Operational monitoring of weather radar receiving chain using the sun, J. Atmos. Ocean. Technol., 27, 159-166, doi:10.1175/2009JTECHA1213.1, 2010.
Huuskonen, A. and Holleman, I.: Determining weather radar antenna pointing using signals detected from the sun at low antenna elevations, J. Atmos. Ocean. Technol., 24, 476-483, 2007.

Kneizys, F. X., Abreu, L. W., Anderson, G. P., Chetwynd, J. H., Shettle, E., Berk, A., Bernstein, L. S., Robertson, D. C., Acharya, P., Rothman, L. S., Selby, J. E. A., Gallery, W. O., and Clough, S.: The MODTRAN 2/3 and the LOWTRAN 7 model, Philips Laboratory Tech. Rep. MA 01731-3010, 1996.

Liebe, H. J., Hufford, G. A., and Gotton, M. G.: Propagation modeling of moist air suspended water/ice particles at frequencies below $1000 \mathrm{GHz}$, in: AGARD-CP-542, Atmospheric Propagation Effects Through Natural and Man-Made Obscurants for Visible to MM-Wave Radiation, Palma de Mallorca, Spain, 1993.

Ollivier, F.: Instruments topographiques, Eyrolles, Paris, France, 1955.

Rees, W. G. and Steel, A. M.: Radar backscatter coefficients and snow detectability for upland terrain in Scotland, Int. J. Remote Sens., 22, 3015-3026, 2001.

Rico-Ramirez, M. A., Gonzalez-Ramirez, E., Cluckie, I., and Han, D.: Real-time monitoring of weather radar antenna pointing using digital elevation and a Bayes clutter classifier, Meteorol. Appl., 16, 227-236, 2009.

Swisstopo: DHM25, the digital height model of Switzerland, Tech. rep., Swisstopo, available at: http://www.swisstopo. admin.ch/internet/swisstopo/en/home/products/height/dhm25. parsysrelated1.44518.downloadList.76700.DownloadFile.tmp/ dhm25infoen.pdf (last access: 2 March 2012), 2004.

Ulaby, F. T., Moore, R. K., and Fung, A. K.: Microwave remote sensing vol. II - Radar remote sensing and surface scattering and emission theory, Artech House, Norwood, MA, USA, iSBN 089006-191-2, 1986.

Whyte, W. S.: Basic metric surveying, Newnes-Butterworths, London, UK, 1969. 